\title{
TD-DFT and TD-DFTB Investigation of the Optical Properties and Electronic Structure of Silver Nanorods and Nanorod Dimers
}

\author{
Fahri Alkan and Christine M. Aikens*(i) \\ Department of Chemistry, Kansas State University, Manhattan, Kansas 66506, United States \\ Supporting Information
}

ABSTRACT: Here, we perform theoretical investigation using time-dependent density functional theory (TD-DFT) and time-dependent density functional tight binding (TDDFTB) for the electronic structure and optical properties of silver nanorods. TD-DFTB generally performs well for the accurate description of optical properties with respect to the size and type of dimer assembly of silver nanorods compared to TD-DFT. However, the energies and intensities of the longitudinal and transverse peaks of the nanorods are somewhat underestimated with TD-DFTB compared to the values calculated at the TD-DFT level. By exploiting the computational efficiency of TD-DFTB, we also extend our investigation to longer nanorods and their dimers containing up to $\sim 2000$ atoms. Our results show that the coupling between nanorods and the resulting optical properties of the dimer assemblies are quite dependent on the length of the monomers. In all cases, the energy shifts in dimers as a function of the gap distance deviate significantly from the dipole-dipole interaction model. Moreover, a comparison of the best-fit curves for the dependence of the fractional shifts $\left(\Delta \lambda / \lambda_{0}\right)$ on nanorod length indicates that the parameters of the plasmon ruler equation depend on the length of the nanorods and the type of the assembly rather than approaching a universal value. These insights are enabled by the computational efficiency of TD-DFTB and its ability to treat quantum mechanical effects in large nanorod dimer systems.

\section{INTRODUCTION}

Noble-metal nanoparticles exhibit unique optical properties associated with their localized surface plasmon resonances (LSPR). ${ }^{1-7}$ The LSPR occurs when the free electrons of the metal are collectively excited by an incident electromagnetic field and is often characterized by a strong absorption in the optical spectrum of the nanoparticle in the UV-vis or near-IR regime. $^{8-10}$ The resonance condition for the plasmon depends strongly on conditions such as particle size, ${ }^{11-13}$ shape, ${ }^{14-16}$ composition, ${ }^{17,18}$ or the dielectric constant of the medium. ${ }^{13}$ Due to this tunability of the LSPR, plasmonic nanoparticles have been investigated for their applications in biosensors, catalysis, nanoantennas, and light-harvesting devices. ${ }^{19-32}$ Additionally, the optical properties of plasmonic nanoparticles also depend on their proximity to other such particles due to plasmonic coupling. ${ }^{33-47}$ In this case, the collective properties of a nanoparticle assembly can be tuned by controlling geometric factors, such as particle-particle distances or symmetry of the assembly. ${ }^{9,48,49}$

For many cases of interest, the optical properties of large nanoparticles can be successfully treated theoretically by solving the classical Maxwell equations using methods such as Mie theory, discrete dipole approximation (DDA), or the finite difference time domain method. ${ }^{38,50-55}$ However, classical treatment of these nanoparticles can be problematic in cases where the quantum mechanical effects become important, such as when the particle dimensions are below the range of 5-10 $\mathrm{nm}^{56}$ or when the gap distances between two nanoparticles are on the subnanometer scale. ${ }^{45,57,58}$ Consequently, ab initio methods, such as time-dependent density functional theory (TD-DFT), have been employed for various types of nanoclusters, where such effects become important. ${ }^{59}$ Unfortunately, regular TD-DFT calculations that employ explicit nuclei and atomic basis sets are typically employed efficiently for systems with up to a few hundred (or occasionally a few thousand) metal atoms due to the high computational cost with increasing number of atoms. Nevertheless, a considerable number of studies have investigated the plasmonic properties of relatively small nanoclusters using TD-DFT methods. ${ }^{60-71}$ Approximate TD-DFT methods, such as simplified TammDancoff approximation ${ }^{72}$ or simplified TD-DFT, ${ }^{73}$ can potentially be applied to larger clusters. A reasonable approximation for TD-DFT calculations involves the use of the jellium model, where the nuclear charges and core electrons are replaced with a background uniform charge. Due to its simplicity, jellium-based TD-DFT calculations have been employed to investigate the quantum effects on the plasmonic

Received: May 30, 2018

Published: June 1, 2018 
properties of larger metallic nanoparticles with success. ${ }^{45,58,74-78}$ We note that the search for theoretical methodologies that can accurately describe quantum effects in large systems with reasonable computational cost is still a very active research area in computational chemistry and physics, ${ }^{79}$ and several reviews exist in the literature that have discussed the limitations and capabilities of different theoretical treatments for plasmonic nanoparticles. ${ }^{7,59,79-81}$

One possible candidate that can account for quantum mechanical effects in large systems is the density functional tight binding (DFTB) method ${ }^{82,83}$ and its time-dependent formalism (TD-DFTB), ${ }^{84,85}$ which aims to achieve the accuracy of DFT methods and the efficiency of tight-binding-based methods. The TD-DFTB method has been applied to predict the optical properties and excited-state electron dynamics of large systems, such as semiconductor nanoparticles, ${ }^{86-88}$ silicon quantum dots, ${ }^{89-92}$ and other nanoscale systems. ${ }^{93-95}$ More recently, Sanchez and co-workers have investigated the relaxation dynamics of LSPR and the effect of oxidation on the plasmonic properties of metal nanoclusters. ${ }^{96-98}$ Their results have shown that the TD-DFTB method can produce the expected size effect for the LSPR of such systems. ${ }^{96}$ In another recent work, Ilawe et al. ${ }^{99}$ utilized the real-time TD-DFTB formalism to understand the electron dynamics of plasmonic antennas. Despite its increasing popularity in recent years, the efficiency and the capabilities of TD-DFTB in terms of how well it can describe quantum mechanical effects in calculations of plasmonic properties for metallic nanoparticles are still not compared to other ab initio methods or classical methods. Therefore, more studies are needed to answer these key questions.

In this work, we investigate the optical properties of small to medium-sized pentagonal silver nanorods and their dimer assemblies using TD-DFT and TD-DFTB methods. For shorter-nanorod systems $\left(\mathrm{Ag}_{19}-\mathrm{Ag}_{67}\right)$, the calculated absorption spectra and electronic structures at the TD-DFTB level are compared to those obtained with TD-DFT for benchmark cases. Furthermore, the absorption spectra of the dimers of silver nanorods are investigated for longer nanorods with TDDFTB to gain more clarity about the effects of length on their optical properties. Moreover, we investigate the effect of gap distance on the absorption spectra for dimers. Our aim is to explore the potential of TD-DFTB methods for the accurate prediction of plasmonic properties for individual nanoparticles and their assemblies.

\section{COMPUTATIONAL METHODS}

Geometries of the Nanorod Monomers and Dimers. All calculations were performed using the ADF2016 package. ${ }^{100-102}$ For small nanorods discussed in the first subsection of Results and Discussion, the geometry of the monomer was optimized using the BP86 functional ${ }^{103,104}$ and a DZ (large core) basis set. This level of theory was previously employed for the geometries of related systems. ${ }^{61}$ For the dimer structure, the relaxed geometries of the monomers were used with the desired particle separation distance. We have analyzed two different assemblies of the monomers: side-by-side (dimer A) and end-to-end (dimer B) configurations. Figure 1 illustrates the two different assemblies of silver nanorod dimers that are investigated in this work. The separation distance between two clusters $(R)$ is regarded as the nearest $\mathrm{Ag}-\mathrm{Ag}$ distance in a given structure. For longer nanorods that are discussed in the second subsection, the geometries of the monomers were

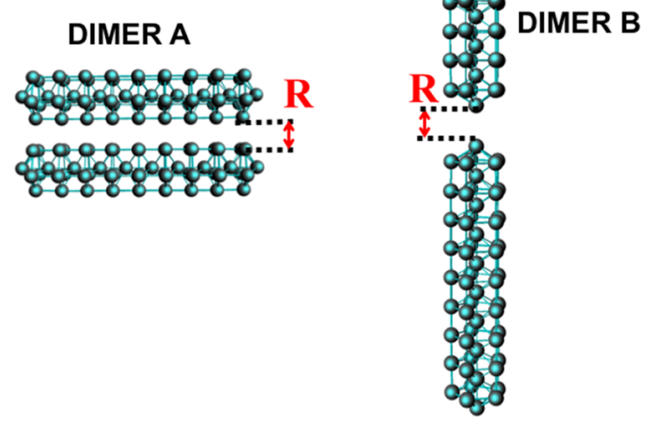

Figure 1. Different dimer assemblies of $\mathrm{Ag}_{55}$ nanorod. Dimer A represents the side-by-side configuration, whereas dimer $B$ represents the end-to-end configuration with separation distance $R$.

generated by using a $\mathrm{Ag}_{6}$ base unit. The coordinates of the base unit are taken from the optimized geometry of the $\mathrm{Ag}_{55}$ nanorod and are provided in the Supporting Information (SI). This unit was repeated along the $z$ axis (i.e., the long axis) of the system by $2.85 \AA$ increments until the desired length was reached for the nanorod. The dimer structures were generated using the same method discussed previously. In this work, all of the nanorods have the same diameter, so longer nanorods consequently have larger aspect ratios.

Absorption Spectra. Excited states of the silver nanorods were calculated using TD-DFT or TD-DFTB methods, as implemented in the ADF2016 package. ${ }^{105,106}$ For the TD-DFT calculations, we employed the Perdew-Burke-Ernzerhof (PBE) density functional ${ }^{107,108}$ and DZ basis set (large core) without any relativistic effects; this level of theory was chosen to compare closely to TD-DFTB calculations that can be viewed as approximations to a PBE calculation with a minimal basis set. TD-DFTB calculations were performed using the selfconsistent-charge DFTB formalism ${ }^{109,110}$ and the Hyb-0-2 parameter set. ${ }^{111,112}$ The absorption spectra of the nanorods were obtained by employing a Gaussian broadening with 0.15 $\mathrm{eV}$ full width at half-maximum. For short nanorods $\left(\mathrm{Ag}_{19}-\right.$ $\left.\mathrm{Ag}_{67}\right)$, the pairs of occupied and virtual orbitals with oscillator strengths of the single-orbital transitions less than $5 \times 10^{-4}$ were eliminated from the guess vector of the excited states. For longer nanorods $\left(\mathrm{Ag}_{91}-\mathrm{Ag}_{985}\right)$, this threshold was raised to $10^{-3}$. Such a threshold is often necessary for large systems, such as dimer assemblies of $\mathrm{Ag}_{799}$ or $\mathrm{Ag}_{985}$, since the number of excited states that must be calculated to obtain a spectrum that covers the range of $0.0-4.0 \mathrm{eV}$ can be $15000-18000$.

Charges on the Nanorods. Due to the odd number of silver atoms in all monomer structures, a closed-shell electron configuration can only be achieved with an odd value for the total charge for monomers and an even value for the total charge for dimers. For all calculations containing $\mathrm{Ag}_{19}-\mathrm{Ag}_{67}$, the charges on the monomers are set to +1 and the charges for the dimers are set to +2 . For longer nanorods, +1 or +2 charges on some systems yielded partial occupations of the orbitals with the TD-DFTB method, which prohibits the calculation of excited states with this approach. For $\mathrm{Ag}_{595}$, the total charges are set to $-1,+2$, and -2 for the monomer, dimer $\mathrm{A}$, and dimer $\mathrm{B}$ systems, respectively. For $\mathrm{Ag}_{799}$, the total charges are set to $-1,-2$, and -2 for the monomer, dimer $\mathrm{A}$, and dimer $\mathrm{B}$ 
systems, respectively. For $\mathrm{Ag}_{985}$, the total charges are set to -1 , 0 , and -2 for the monomer, dimer $\mathrm{A}$, and dimer B systems, respectively. For the remaining systems, the total charges are set to +1 for monomers and +2 for both dimer assemblies. We also note that the variation of the total charge between +2 and -2 has a minimal impact on the calculated absorption spectra, especially for longer nanorods. A comparison of the calculated spectra is shown in the SI for the dimer A structure of $\mathrm{Ag}_{391}$ with +2 and -2 total charges.

\section{RESULTS AND DISCUSSION}

Comparison of TD-DFT and TD-DFTB for the Absorption Spectra of Silver Nanorod Monomers and Dimers. Monomers. In the first section, we compare the performances of TD-DFT and TD-DFTB for the calculated absorption spectra of monomer silver nanorods with varying lengths. Figure 2a shows the calculated absorption spectra for
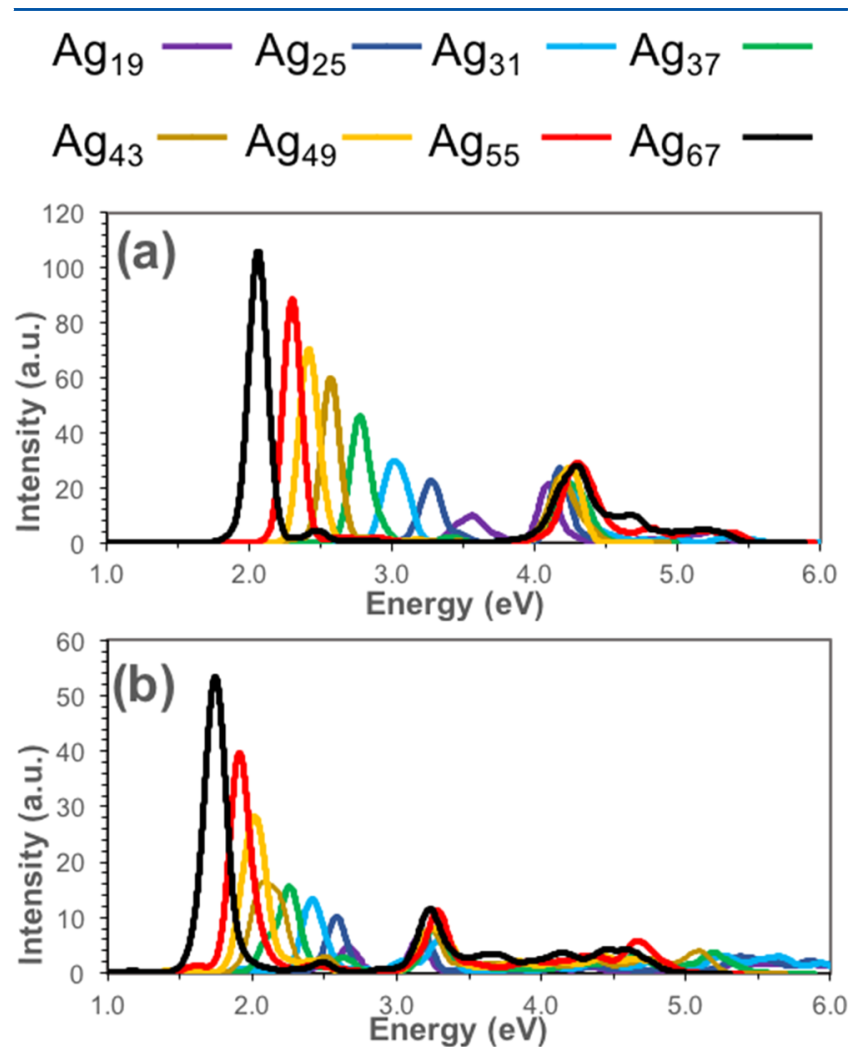

Figure 2. Calculated absorption spectra for silver nanorods $\left(\mathrm{Ag}_{19}\right.$, $\mathrm{Ag}_{25}, \mathrm{Ag}_{31}, \mathrm{Ag}_{37}, \mathrm{Ag}_{43}, \mathrm{Ag}_{49}, \mathrm{Ag}_{55}$, and $\mathrm{Ag}_{67}$ ) at the (a) TD-DFT and (b) TD-DFTB levels of theory.

these monomers $\left(\mathrm{Ag}_{19}, \mathrm{Ag}_{25}, \mathrm{Ag}_{31}, \mathrm{Ag}_{37}, \mathrm{Ag}_{43}, \mathrm{Ag}_{49}, \mathrm{Ag}_{55}\right.$, and $\left.\mathrm{Ag}_{67}\right)$ at the TD-DFT level of theory, whereas Figure $2 \mathrm{~b}$ shows the same spectra calculated with the TD-DFTB level of theory. Previous TD-DFT investigations ${ }^{18,61,63}$ on the silver nanorods have indicated that the absorption spectra of similar systems mainly exhibit two spectral features: a low-energy longitudinal peak and a high-energy transverse peak. As shown in Figure 2a, our TD-DFT results at the PBE/DZ level of theory exhibit a similar spectral shape compared to previous findings ${ }^{18,61,63}$ for silver nanorods, as expected. The longitudinal peak red-shifts and becomes more intense with increasing length (or aspect ratio) of the nanorod, whereas the transverse peak slightly shifts to higher energies while slightly gaining intensity. In comparison, the calculated absorption spectra at the TDDFTB level show similar features for the same silver nanorods. As seen from the comparison of the two spectra shown in Figure 2a,b, TD-DFTB can generally produce these trends for the energies and the intensities of the peaks.

For a more quantitative comparison of the two methods, we tabulate the energy and the intensity for the maxima of the longitudinal and transverse peaks in Table 1 for the spectra shown in Figure 2a,b. For both peaks, the positions of the maxima are predicted at lower energies using the TD-DFTB level compared to those obtained at the TD-DFT level of theory. At the TD-DFT level, the longitudinal peak red-shifts by $1.52 \mathrm{eV}$ as the size of the system increases from $\mathrm{Ag}_{19}$ to $\mathrm{Ag}_{67}$. The same redshift is calculated to be $0.93 \mathrm{eV}$ with TD-DFTB. In addition, the predicted intensities are systematically lower for the spectra calculated with TD-DFTB. From $\mathrm{Ag}_{19}$ to $\mathrm{Ag}_{67}$, the intensity of the longitudinal peak exhibits a 10 -fold increase at the TD-DFT level, which is also the case at the TD-DFTB level. However, in general, the calculated intensities of the longitudinal peak with TD-DFTB are roughly half of the intensities obtained from TD-DFT calculations, except for $\mathrm{Ag}_{37}$ and $\mathrm{Ag}_{43}$, where the maximum peak intensities calculated by TD-DFTB are even lower. In these cases, the longitudinal peaks originate dominantly from a single excited state with high oscillator strength in the case of TD-DFT. In comparison, multiple excited states contribute to the longitudinal peaks of $\mathrm{Ag}_{37}$ and $\mathrm{Ag}_{43}$ in the case of TD-DFTB, which lowers the predicted maximum intensities of the peaks due to the broadening observed.

As the system size increases, the difference in the predicted energies of the longitudinal peak between the TD-DFT and TD-DFTB levels of theory $(\Delta E)$ monotonically decreases with increasing length. $\Delta E$ for the longitudinal peak varies between 0.89 and $0.30 \mathrm{eV}$ for the investigated silver nanorods. In the case of the transverse peak, the changes in the energy and intensity with respect to the nanorod length are less pronounced at both levels. $\Delta E$ of the transverse peak varies between 0.94 and $1.06 \mathrm{eV}$ from $\mathrm{Ag}_{19}$ to $\mathrm{Ag}_{67}$, which shows a slight increase with increasing system length. The underestimation of the longitudinal and transverse peak energies with TD-DFTB compared to TD-DFT is predominantly caused by the difference in the predicted electronic structures between the two methods. In general, the energy differences between occupied and unoccupied levels $\left(\varepsilon_{\mathrm{i}}-\varepsilon_{\mathrm{a}}\right)$ are underestimated in calculated excitation energies with TD-DFTB compared to the TD-DFT case for the nanorods.

Dimers. In this section, we extend our investigation to the calculated absorption spectra of dimer nanorods in the arrangements illustrated in Figure 1. Previous experimental and theoretical studies on nanorod dimers have shown that the longitudinal polarization for dimer $\mathrm{A}$ assemblies exhibits a blueshift compared to the monomer peaks, whereas the longitudinal polarization for dimer $\mathrm{B}$ assemblies shows a redshift. ${ }^{9,38,43,44,48,64}$ These results have been often rationalized by the dipole-dipole interaction model; according to this model, the repulsive coupling of the longitudinal polarization and the attractive coupling of the longitudinal polarization lead to dipole-allowed transitions for dimer $\mathrm{A}$ and dimer $\mathrm{B}$ assemblies, respectively. ${ }^{48,113}$ As a result, the longitudinal peak exhibits a blueshift for dimer A and a redshift for dimer $\mathrm{B}$ assemblies of nanorods.

In Figure 3, we compare the calculated spectra of these dimers at $0.4 \mathrm{~nm}$ separation distance using the TD-DFT 
Table 1. Comparison of the TD-DFT and TD-DFTB Methods for the Calculated Energies of the Maxima and Intensities of the Longitudinal and Transverse Peaks of Silver Nanorods

\begin{tabular}{|c|c|c|c|c|c|c|c|c|c|c|}
\hline \multirow[b]{3}{*}{ system } & \multicolumn{5}{|c|}{ longitudinal peak } & \multicolumn{5}{|c|}{ transverse peak } \\
\hline & \multicolumn{2}{|c|}{ TD-DFT } & \multicolumn{2}{|c|}{ TD-DFTB } & \multirow[b]{2}{*}{$\Delta E^{b}(\mathrm{eV})$} & \multicolumn{2}{|c|}{ TD-DFT } & \multicolumn{2}{|c|}{ TD-DFTB } & \multirow[b]{2}{*}{$\Delta E^{b}(\mathrm{eV})$} \\
\hline & energy $(\mathrm{eV})$ & intensity $\left(\mathrm{au}^{a}\right)$ & energy $(\mathrm{eV})$ & $\overline{\text { intensity }(\mathrm{au})}$ & & energy $(\mathrm{eV})$ & $\overline{\text { intensity }(\mathrm{au})}$ & energy $(\mathrm{eV})$ & $\overline{\text { intensity }(\mathrm{au})}$ & \\
\hline $\mathrm{Ag}_{19}$ & 3.56 & 10 & 2.67 & 5 & 0.89 & 4.09 & 21 & 3.15 & 6 & 0.93 \\
\hline $\mathrm{Ag}_{25}$ & 3.27 & 23 & 2.58 & 10 & 0.69 & 4.17 & 27 & 3.18 & 7 & 0.99 \\
\hline $\mathrm{Ag}_{31}$ & 3.01 & 30 & 2.41 & 13 & 0.60 & 4.23 & 23 & 3.30 & 6 & 0.93 \\
\hline $\mathrm{Ag}_{37}$ & 2.77 & 46 & 2.26 & 16 & 0.51 & 4.24 & 21 & 3.25 & 8 & 0.99 \\
\hline $\mathrm{Ag}_{43}$ & 2.57 & 60 & 2.08 & 16 & 0.48 & 4.19 & 24 & 3.23 & 8 & 0.96 \\
\hline $\mathrm{Ag}_{49}$ & 2.41 & 70 & 2.02 & 28 & 0.39 & 4.24 & 28 & 3.25 & 8 & 0.99 \\
\hline $\mathrm{Ag}_{55}$ & 2.30 & 89 & 1.91 & 40 & 0.38 & 4.31 & 29 & 3.28 & 11 & 1.02 \\
\hline $\mathrm{Ag}_{67}$ & 2.04 & 106 & 1.74 & 54 & 0.29 & 4.29 & 28 & 3.23 & 12 & 1.06 \\
\hline
\end{tabular}
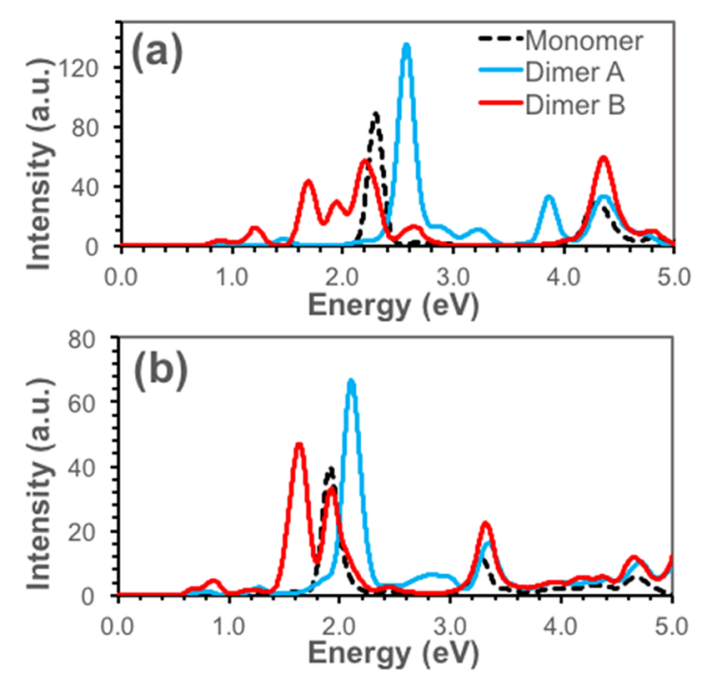

Figure 3. Calculated absorption spectra for different assemblies of $\mathrm{Ag}_{55}$ nanorod dimers and their comparison with monomer spectra at the (a) TD-DFT and (b) TD-DFTB levels of theory.

(Figure 3a) and TD-DFTB (Figure 3b) levels of theory. At this interparticle distance, significant overlap between the wave functions of the monomers is expected; therefore, quantum mechanical effects play an important role in the excited states of these systems. For dimer A, TD-DFT mainly predicts a single feature with a large intensity for the longitudinal peak. This peak exhibits a blueshift of $0.28 \mathrm{eV}$ as expected compared to the case of the monomer nanorod at the same level of theory. ${ }^{43,113}$ On the other hand, the transverse peak strongly splits into two peaks separated by about $0.50 \mathrm{eV}$ compared to the spectrum of the monomer. The splitting of the transverse peak is a result of symmetry breaking of the transverse polarization (which is polarized in $x$ and $y$ directions), dictated by the symmetry group $\left(C_{2 v}\right)$ of dimer A. At the TD-DFTB level of theory, a blueshift is also predicted for the longitudinal peak; however, this blueshift is underestimated by $0.09 \mathrm{eV}$ compared to the TD-DFT case. Splitting of the transverse peak is also observed at the TD-DFTB level, resulting in two features in the 2.5-3.5 $\mathrm{eV}$ region. The high-energy feature is sharp and exhibits a slight blueshift compared to the transverse peak of the monomer, similar to the case at the TD-DFT level of theory. However, the low-energy feature is significantly broader with TD-DFTB compared to the spectrum obtained at the TD-DFT level.

In the case of dimer B, calculated spectra at the TD-DFT level exhibit multiple features for the longitudinal peaks, which are mainly red-shifted compared to the single peak observed in the monomer spectra. We also note that the additional bands for the longitudinal polarization have been shown for the dimer B assembly of nanorods previously in both experimental and theoretical works. ${ }^{38,43,64}$ The splitting of the longitudinal peak is also observed at the TD-DFTB level. However, there are some differences in the number of peaks and the strength of the redshifts between the spectra predicted at the TD-DFT and TD-DFTB levels of theory. In comparison, a single transverse peak, which gains intensity compared to the monomer spectrum, is calculated by both methods. In both cases, the transverse peak shows a slight blueshift $(0.05$ and $0.04 \mathrm{eV}$ for TD-DFT and TD-DFTB, respectively) compared to the case of the monomer nanorod. Overall, there is a qualitative agreement between the TD-DFT and TD-DFTB levels of theory for the spectra of monomer and dimer silver nanorods. A similar agreement between the two methods is also seen for the predicted electronic structures and the configuration of the excited states that contributes to the absorption spectra of the investigated monomer and dimer nanorods. A comparison of ground-state and excited-state electronic structure is given in the SI for $\mathrm{Ag}_{55}$ monomer and dimers.

Distance Dependence of the Absorption Spectra. In Figure $4 a$, we show the correlation between the blueshift of the maximum for the longitudinal peak $\left(\Delta E_{\text {blueshift }}=\mid E_{\text {dimer }}-\right.$

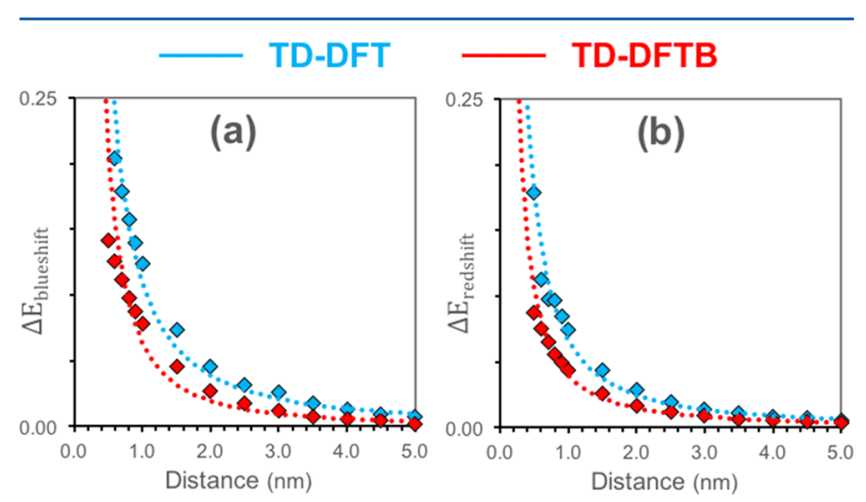

Figure 4. Correlation between (a) $\Delta E_{\text {blueshift }}$ and (b) $\Delta E_{\text {redshift }}$ and gaps for dimer $\mathrm{A}$ and dimer $\mathrm{B}$ assemblies of $\mathrm{Ag}_{55}$ nanorods calculated with the TD-DFT and TD-DFTB levels of theory. The dotted lines show the best-fit curve that follows the form $\Delta E=A R^{-n}$. For $\Delta E_{\text {blueshift }}$ vs $R$, $A$ and $n$ parameters of the best fit are 0.11 and 1.51 for TD-DFT and 0.06 and 1.73 for TD-DFTB, respectively. For $\Delta E_{\text {redshift }}$ vs $R, A$ and $n$ parameters of the best-fit curve are 0.07 and 1.43 for TD-DFT and 0.04 and 1.41 for TD-DFTB, respectively. 
$\left.E_{\text {monomer }} l\right)$ and the separation distance $(R)$ of the nanorods for the dimer A assembly. In comparison, Figure $4 \mathrm{~b}$ shows the same comparison for the redshift of the longitudinal peak $\left(\Delta E_{\text {redshift }}=\left|E_{\text {dimer }}-E_{\text {monomer }}\right|\right)$ for the dimer B assembly. For both methods, the longitudinal peak of the dimer monotonically shifts to higher energies as the separation distances between the nanorods increase for dimer $\mathrm{A}$ and shifts to lower energies in the same direction for dimer B as expected from the previous work on nanorods. At small gaps $(0.4-1.0 \mathrm{~nm})$, $\Delta E_{\text {blueshift }}$ of the longitudinal peak is underestimated with TDDFTB compared to the TD-DFT case. This is also the case for $\Delta E_{\text {redshift }}$ for dimer $\mathrm{B}$. This underestimation at small gap distances is due to the fact that the coupling between the monomer-based orbitals is predicted to be smaller with DFTB and TD-DFTB. The deviation between the two methods for the overlap of monomeric levels in the $0.4-1.0 \mathrm{~nm}$ range is most likely a result of the minimal basis nature of the DFTB formalism, which often exhibits an overestimation for the orbital coupling for shorter gaps (ca. $0.2-0.4 \mathrm{~nm}$ ) and an underestimation at longer range (ca. $0.4-1.0 \mathrm{~nm})$ compared to the PBE/DZ level of theory. This is demonstrated for the AgAg dimer in the SI, where we provide a detailed comparison of the two methods for the predicted bonding energies and orbital interactions. As the gaps increase, the deviations between the two methods for the predicted $\Delta E_{\text {blueshift }}$ and $\Delta E_{\text {redshift }}$ become smaller. Additionally, the calculated intensities of the longitudinal peak become larger as the gaps increase with both methods. At a separation distance of $5.0 \mathrm{~nm}$, both methods predict that the blueshift of the longitudinal peak of the dimer vanishes, whereas the peak intensity doubles compared to the spectra of the monomer system, indicating that the two monomers are essentially uncoupled at this distance.

Under the assumptions that the interaction between the excited states of two nanorods is a weak perturbation to the Hamiltonian and that this term can be expressed as a dipoledipole interaction, both $\Delta E_{\text {blueshift }}$ and $\Delta E_{\text {redshift }}$ are expected to be proportional to $|\mu|^{2} R^{-3}$, where $\mu$ is the dipole moment of the excited state for the monomer and $R$ is the distance between two dipoles. In Figure 4, we also show the best-fit curve in the form of $A R^{-n}$ for the calculated $\Delta E_{\text {blueshift }}$ and $\Delta E_{\text {redshift }}$ at the TD-DFT and TD-DFTB levels. The best-fit curves in both cases do not follow the $A R^{-3}$ relationship, which is expected because the dipole-dipole interaction relation does not hold particularly at short gaps. Additionally, due to the finite size of nanorods, the best-fit relation depends on our definition of $R$ for these systems. When $R$ is considered as the nearestneighbor distance, as shown in Figure 1, TD-DFT and TDDFTB, respectively, predict $n$ to be 1.51 and 1.73 for dimer A and 1.43 and 1.41 for dimer $B$ for the range $0.5-5.0 \mathrm{~nm}$, clearly showing a deviation from the $R^{-3}$ relation.

Length Dependence of Optical Properties for LongerNanorod Monomers and Dimers. Monomers of Longer Nanorods. In this section, we extend our investigation to longer nanorods $\left(\mathrm{Ag}_{91}, \mathrm{Ag}_{199}, \mathrm{Ag}_{391}, \mathrm{Ag}_{595}, \mathrm{Ag}_{7999}\right.$, and $\left.\mathrm{Ag}_{985}\right)$ and the dimer $\mathrm{A}$ and $\mathrm{B}$ assemblies of these nanorods. The calculations with these systems only utilize the TD-DFTB formalism as TD-DFT methods cannot be employed efficiently for dimers across this size range. In Figure $5 \mathrm{a}, \mathrm{b}$, we show the longitudinal and transverse peaks, respectively, in the absorption spectra of monomer nanorods. Table 2 shows the energies and the intensities of the maxima for the longitudinal and transverse peaks.
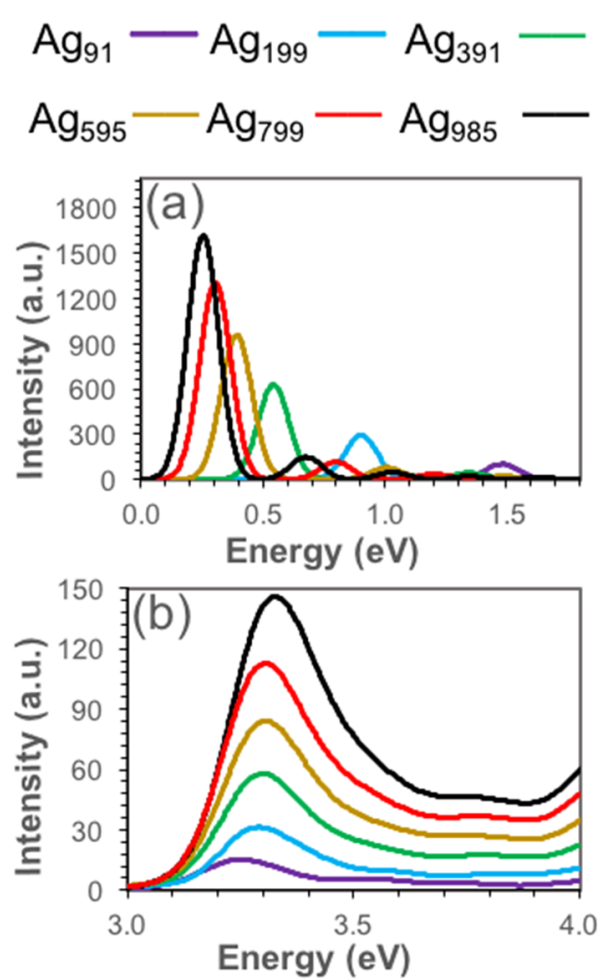

Figure 5. Calculated absorption spectra for longer silver nanorods at the TD-DFTB level of theory. (a) Longitudinal peak for various lengths of nanorods. (b) Transverse peak for the same systems.

Table 2. Calculated Energies of the Maxima and Intensities of the Longitudinal and Transverse Peaks of Longer Silver Nanorods Using TD-DFTB

\begin{tabular}{lccccc} 
& \multicolumn{2}{c}{ longitudinal peak } & & \multicolumn{2}{c}{ transverse peak } \\
\cline { 2 - 3 } \cline { 5 - 6 } system & energy $(\mathrm{eV})$ & intensity $(\mathrm{au})$ & & energy $(\mathrm{eV})$ & intensity $(\mathrm{au})$ \\
$\mathrm{Ag}_{91}$ & 1.48 & 101 & & 3.25 & 15 \\
$\mathrm{Ag}_{199}$ & 0.90 & 295 & & 3.29 & 31 \\
$\mathrm{Ag}_{391}$ & 0.54 & 626 & & 3.30 & 58 \\
$\mathrm{Ag}_{595}$ & 0.39 & 957 & & 3.31 & 84 \\
$\mathrm{Ag}_{799}$ & 0.30 & 1305 & & 3.30 & 113 \\
$\mathrm{Ag}_{985}$ & 0.25 & 1618 & & 3.32 & 146 \\
\hline
\end{tabular}

Similar to the smaller nanorods shown in Figure 2, the longitudinal peak for the longer nanorods also displays a strong redshift with increasing length of the nanorod. This redshift shows a convergent behavior, and the energy of the longitudinal peak can be fitted to a functional form of $A L^{-b}\left(R^{2}=0.997\right)$, where $b=0.74, A=4.54$, and $L$ is the length of the nanorod as calculated from the tip-to-tip distance. As the length of the nanorod increases, the longitudinal peak gains intensity as well. The increase in the intensity follows a linear relation, where the best-fit line equation is described as $y=34.3 L$ with $R^{2}=0.998$. In comparison, the maximum of the transverse peak shows a slight blueshift with increasing length of the nanorods. The blueshift of the transverse peak is very small for the investigated systems, amounting to only $0.07 \mathrm{eV}$ between the shortest and longest $\left(\mathrm{Ag}_{91}\right.$ and $\left.\mathrm{Ag}_{985}\right)$ nanorods. In comparison, the redshift of the longitudinal peak between the same two systems is 1.23 $\mathrm{eV}$. The energy of the transverse peak can be fitted to a functional form of $A L^{-b}$ as well with $b=0.008$, although the fit is not as good $\left(R^{2}=0.893\right)$ compared to the case for the longitudinal peak. On the other hand, there is a good 
correlation between the intensity of the transverse peak and the length of the nanorod, which follows the relation $y=3.06 \mathrm{~L}$ with $R^{2}=0.998$. It is seen that the gain in intensity with increasing length of the nanorod is $\sim 10$ times smaller for the transverse peak compared to the longitudinal peak, as indicated by the comparison of the slopes of the linear correlations. The correlations between the energies and the intensities of the peaks with the lengths of the nanorods along with the best-fit curves are shown in the SI.

Dimers of Longer Nanorods. In Figure 6a-f, the longitudinal peaks in the absorption spectra of dimer A and
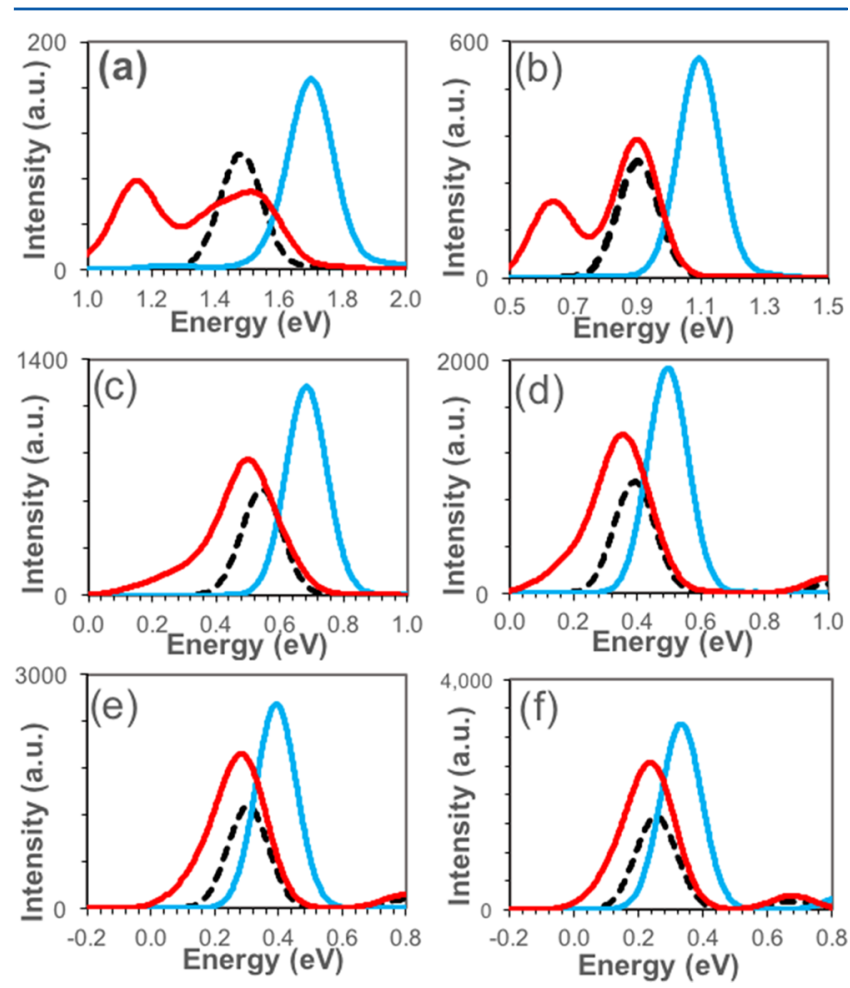

Figure 6. Calculated longitudinal peaks for the dimers of (a) $\mathrm{Ag}_{91}$, (b) $\mathrm{Ag}_{199}$, (c) $\mathrm{Ag}_{391}$, (d) $\mathrm{Ag}_{595}$, (e) $\mathrm{Ag}_{799}$, and (f) $\mathrm{Ag}_{985}$. The dotted line shows the monomer spectrum whereas the blue and red solid lines represent the spectra for dimer A and dimer B assemblies, respectively.

$\mathrm{B}$ assemblies are compared to the peaks of the monomer for the $\mathrm{Ag}_{91}, \mathrm{Ag}_{199}, \mathrm{Ag}_{391}, \mathrm{Ag}_{595}, \mathrm{Ag}_{799}$, and $\mathrm{Ag}_{985}$ nanorods. The interparticle separation distance of the nanorods is $0.4 \mathrm{~nm}$ for all systems.

In the case of dimer $\mathrm{A}$, the spectra for all of the investigated systems mainly exhibit a single feature for the longitudinal peak, which shows a blueshift compared to the monomer peak. This blueshift is largest for the dimer of $\mathrm{Ag}_{91}(0.22 \mathrm{eV})$, and systematically decreases with increasing length of the nanorod. The blueshift of the longitudinal peak is only $0.08 \mathrm{eV}$ for the longest nanorod investigated, $\mathrm{Ag}_{985}$. In the case of the dimer $\mathrm{B}$ assembly, there is considerable change observed in the spectral shape with respect to increasing length of the nanorods. For $\mathrm{Ag}_{91}$, the longitudinal peak mainly splits into two features. The maximum of the first peak shows a redshift of $0.33 \mathrm{eV}$, whereas the second peak displays a slight blueshift compared to the monomer spectra. For $\mathrm{Ag}_{199}$, the peak at the higher energy strongly gains intensity, similar to the single peak observed in dimer A, whereas the intensity of the peak at lower energy mainly remains unchanged. Additionally, the energy difference between the maxima of these peaks decreases with increasing length of the nanorod. As a result, the splitting of the longitudinal peak is not as pronounced in the spectra for the dimers of longer nanorods, such as $\mathrm{Ag}_{799}$ or $\mathrm{Ag}_{985}$.

In Figure $7 \mathrm{a}-\mathrm{f}$, we show the transverse peaks in the absorption spectra of dimer A and B assemblies along with the
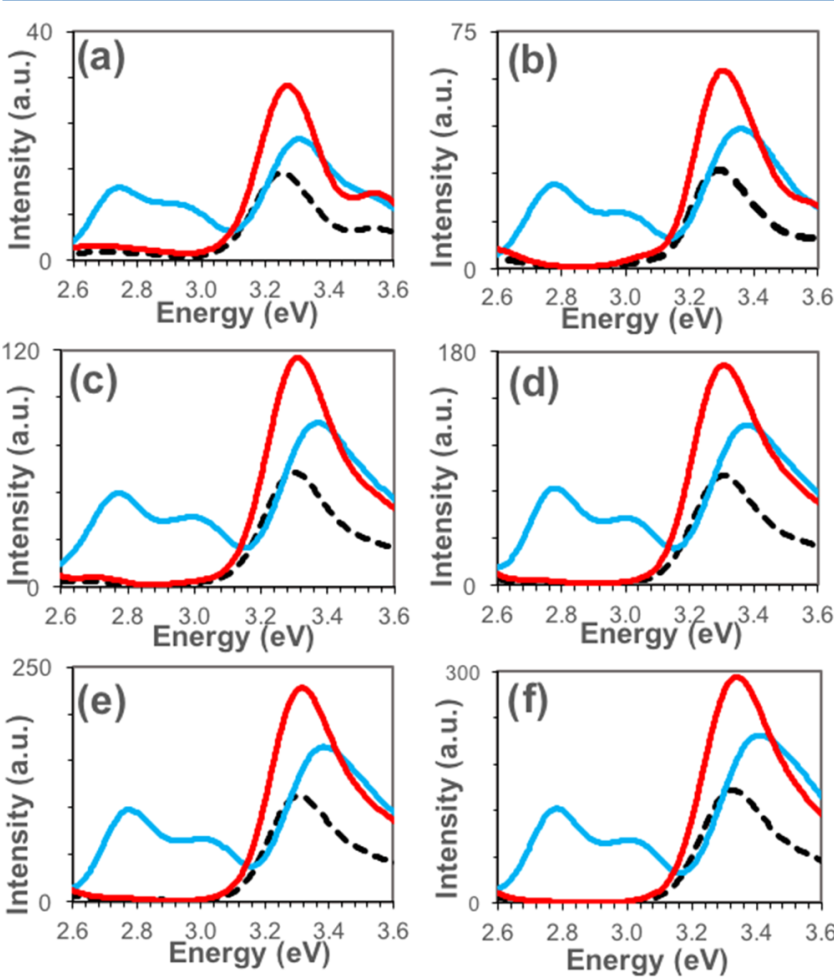

Figure 7. Calculated transverse peaks for the dimers of (a) $\mathrm{Ag}_{91}$, (b) $\mathrm{Ag}_{199}$, (c) $\mathrm{Ag}_{391}$, (d) $\mathrm{Ag}_{595}$, (e) $\mathrm{Ag}_{799}$, and (f) $\mathrm{Ag}_{985}$. The dotted line shows the monomer spectra, whereas the blue and red solid lines represent the spectra for dimer A and dimer B assemblies, respectively.

monomer spectra for the same nanorod systems. Unlike the longitudinal peak, the spectral shape and the energetics of the transverse peak for the dimers show very little dependence on the length of the nanorods. For all systems, the transverse peak splits into multiple features in the case of dimer A, whereas a single feature is observed for dimer B assembles. These results are also in agreement with our previous findings at both the TD-DFT and TD-DFTB levels of theory for smaller nanorods, as shown in Figure 3.

The only main change for the transverse peaks of the dimers as the length of the nanorods increases is an increase in the intensities of the peaks, which is also observed in the individual monomers (Table 2). The different trends observed for the effects on the longitudinal and transverse peaks upon dimerization of the nanorods are related to the fact that the investigated systems vary only in length, i.e., in the polarization axis $(z)$ of the longitudinal peak, whereas the width of the nanorods (polarization axes $(x, y)$ of the transverse peak) is fixed. As a result, we observe very similar splitting profiles and relative energy shifts for the transverse peaks of the dimers. A future investigation will address how these properties of the dimer spectra differ with variation in the width of the nanorod.

Distance Dependence of the Longitudinal Peak in LongerNanorod Dimers. In Figure $8 \mathrm{a}-\mathrm{f}$, we show the distribution of longitudinal peaks for varying separation distances (0.4-5.0 

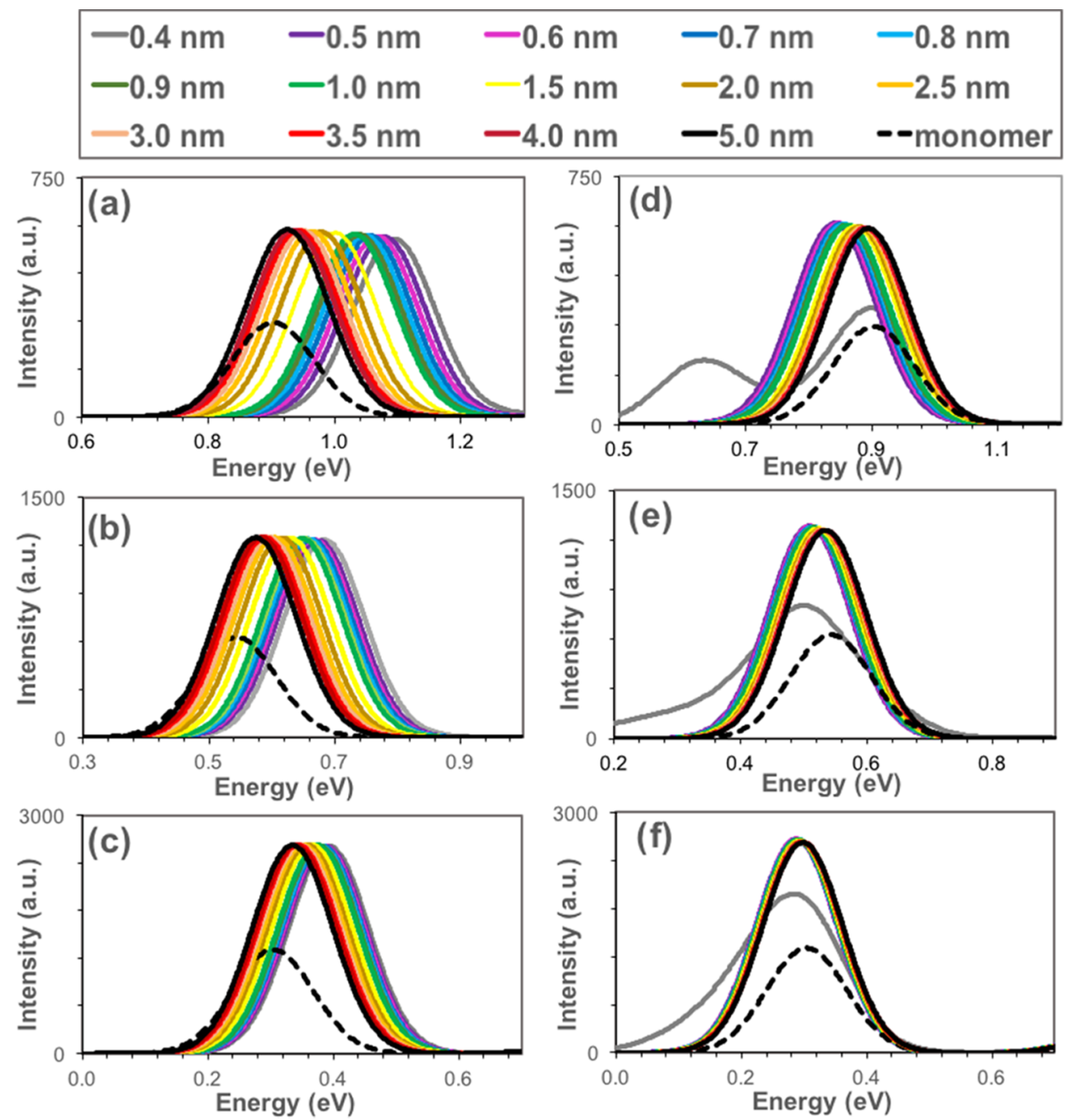

Figure 8. Calculated longitudinal peaks of the dimers of $\mathrm{Ag}_{199}, \mathrm{Ag}_{391}$, and $\mathrm{Ag}_{799}$ for separation distances 0.4-5.0 nm. (a-c) Spectra of the dimer $\mathrm{A}$ assembly for $\mathrm{Ag}_{199}, \mathrm{Ag}_{391}$, and $\mathrm{Ag}_{799}$, respectively. (d-f) Spectra of the dimer $\mathrm{B}$ assembly for $\mathrm{Ag}_{199}, \mathrm{Ag}_{391}$, and $\mathrm{Ag}_{799}$ respectively.

$\mathrm{nm}$ ) in the case of dimer $\mathrm{A}$ (Figure $8 \mathrm{a}-\mathrm{c}$ ) and dimer B (Figure 8d-f) assemblies of $\mathrm{Ag}_{199}, \mathrm{Ag}_{391}$, and $\mathrm{Ag}_{799}$ nanorods. For both types of assemblies, we note that the distribution of peak positions covers a smaller energy range as the nanorod length increases. As the separation distance increases, the maxima of the peaks become closer to the maximum of the monomer peak, as expected. For the dimer $\mathrm{A}$ assembly, the longitudinal peaks still show a slight $\Delta E_{\text {blueshift }}(\sim 0.03 \mathrm{eV})$ compared to the monomer peaks for the nanorods, as shown in Figure 8. At a $15.0 \mathrm{~nm}$ separation distance (not shown in Figure 8), the blueshifts become less than $0.01 \mathrm{eV}$ for all nanorods.

In the case of dimer $\mathrm{B}$, the longitudinal peak shows significant broadening or splitting at $0.4 \mathrm{~nm}$ separation, as already discussed previously for the TD-DFT and TD-DFTB comparison of $\mathrm{Ag}_{55}$ dimers. In comparison, a single Gaussian feature is predicted for this peak starting from $0.5 \mathrm{~nm}$. Except for the $0.4 \mathrm{~nm}$ separation distance, we note that dimers exhibit a considerably smaller range of longitudinal peak energies for dimer B assemblies compared to their dimer A counterparts. Additionally, at $5.0 \mathrm{~nm}$ separation, the redshifts of the longitudinal peak for dimer $\mathrm{B}$ are below $0.01 \mathrm{eV}$ for all three nanorods, indicating that the interactions between nanorods are smaller for dimer B compared to dimer A, especially for longer separation distances.
In Figure 9a,b, we plot the dependence of $\Delta E_{\text {blueshift }}$ and $\Delta E_{\text {redshift }}$ on separation distance $(0.5-5.0 \mathrm{~nm})$ for dimer $\mathrm{A}$ and dimer $B$ assemblies of the nanorods, respectively. The correlation between the blueshift or redshift and the separation distance follows a similar trend for both assemblies. One main difference, however, is the range of the shifts in the peak position, as discussed previously. For both assemblies, the distance dependence of the blueshift or redshift can be fitted to a function of form $A R^{-b}$, where $b=3$ is expected for a system that is well described by classical dipole-dipole interactions. We note that the goodness of fit is slightly better for the correlation obtained for dimer B compared to the same correlations obtained for the dimer A assemblies. In both cases, the values of $A$ and $b$ become smaller with increasing length of the nanorod. For dimer A, calculated $b$ values from the best-fit curves are 0.808, 0.601, and 0.405 for the dimers of $\mathrm{Ag}_{199}$, $\mathrm{Ag}_{391}$, and $\mathrm{Ag}_{799}$ nanorods, respectively. In comparison, the calculated $b$ values for dimer $\mathrm{B}$ assemblies of $\mathrm{Ag}_{199}, \mathrm{Ag}_{391}$, and $\mathrm{Ag}_{799}$ are $0.858,0.620$, and 0.431 , respectively, which indicates that the $R$ dependence of the energy shifts are predicted to be quite similar in these nanoparticle assemblies.

For all systems, calculated $b$ values deviate significantly from 3 , and this deviation becomes larger with increasing nanorod length. We also note that the correlations in Figure 9 can be 

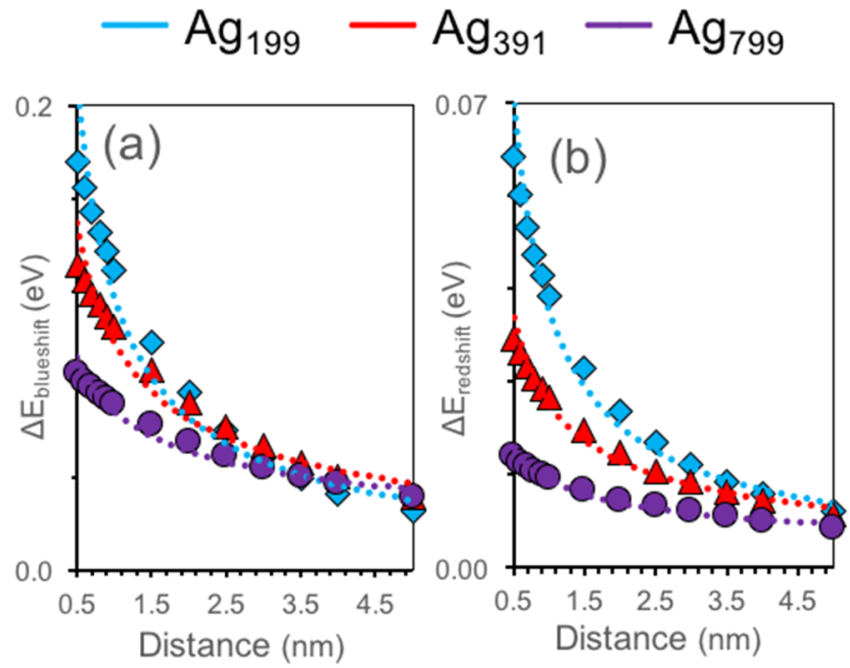

Figure 9. Predicted distance dependence of (a) $\Delta E_{\text {blueshift }}$ in the case of dimer $\mathrm{A}$ assemblies and (b) $\Delta E_{\text {redshift }}$ in the case of dimer $\mathrm{B}$ assemblies of $\mathrm{Ag}_{199}, \mathrm{Ag}_{391}$, and $\mathrm{Ag}_{799}$ nanorods. The dotted line represents the best-fit curve in the form of $\Delta E=A R^{-b}$. The parameters of the best-fit are given in Table 3.

fitted to an exponential function $\left(A \mathrm{e}^{-R b}\right)$ as well. In this case, the goodness of fit becomes better for dimer $\mathrm{A}\left(R^{2}\right.$ is between 0.992 and 0.987$)$, whereas it slightly worsens for dimer B $\left(R^{2}\right.$ is between 0.977 and 0.975 ). The obtained $b$ values from the exponential curves are also predicted to be quite similar for dimer A and B assemblies of each nanorod. In Table 3, we summarize the parameters of the various fits for the distance and length dependence of blueshift or redshift energies for the longitudinal peak.

The distance dependence of plasmon coupling in nanoparticles has also been described by the plasmon ruler model (eq 1) proposed by Jain et al., ${ }^{38,39}$ which relates the fractional shift, or $\Delta \lambda / \lambda_{0}\left(\Delta \lambda\right.$ and $\lambda_{0}$ are the change in excitation wavelength and the monomer excitation wavelength in nanometer), to the separation distance between the nanoparticles with an exponential decay.

$$
\frac{\Delta \lambda}{\lambda_{0}}=A \mathrm{e}^{-(R / L) / \tau}
$$

In eq $1, R$ represents the separation distance, $L$ is the length of the nanorod, and $A$ and $\tau$ are constants of the exponential decay that are determined by the fit. In Figure 10a,b, we show the distance dependence of fractional shifts predicted by TD-

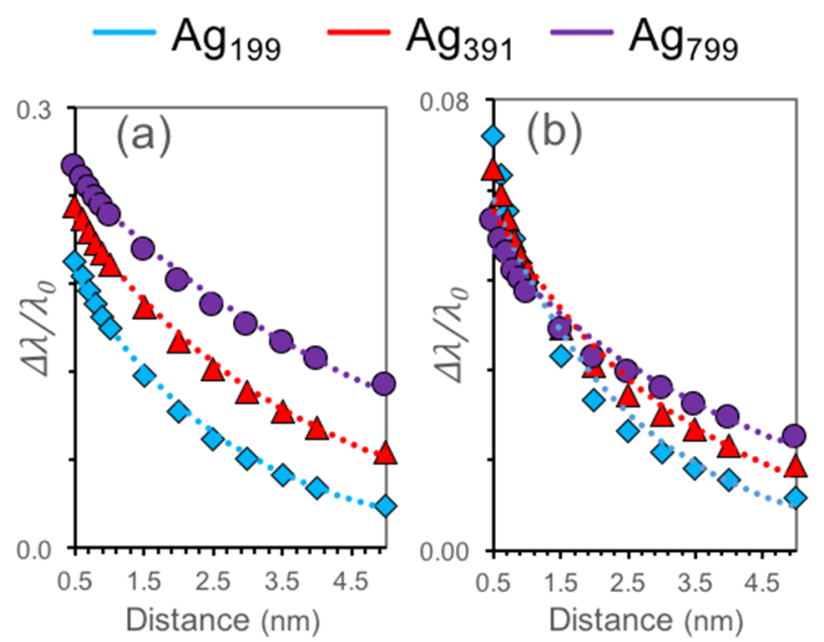

Figure 10. Predicted distance dependence of the fractional shifts for (a) dimer $\mathrm{A}$ assemblies and (b) dimer $\mathrm{B}$ assemblies of $\mathrm{Ag}_{199}, \mathrm{Ag}_{391}$, and $\mathrm{Ag}_{799}$ nanorods. The dotted line represents the best-fit curve in the form of the plasmon ruler equation given in eq 1 .

DFTB for the longitudinal peak of dimer A and dimer B assemblies, respectively. The parameters of the fit are also tabulated in Table 3. Similar to the case for the distance dependence of blueshift and redshift energies, the goodness of fit for the distance dependence of fractional shifts is somewhat better for dimer A assemblies as the $R^{2}$ values are $0.992-0.996$ compared to the goodness of fit for the dimer B assemblies, where the $R^{2}$ values are $0.973-0.975$. In both cases, the $A$ and $\tau$ values predicted by TD-DFTB show a strong dependence on the length of the nanorod and the type of the assembly. For dimer $\mathrm{A}$, the value of $A$ shows an increase with increasing length of the nanorods, whereas it decreases with increasing length of the nanorods in the case of dimer B. In comparison, the predicted value of $\tau$ decreases for both assemblies with increasing length of the nanorod. We also note that the predicted values for $\tau$ are somewhat larger for dimer A compared to the predicted values of the same parameter for dimer B.

The correlation between fractional shifts and the separation distance shown in eq 1 has been investigated experimentally by employing DDA calculations for nanoparticles of different sizes, shapes, or types of metal. ${ }^{38,39,43,44,46,48,114}$ Although the predictions of TD-DFTB for the values of $A$ and $\tau$ are generally in good agreement with previously obtained values $(\tau$ $\sim 0.2$ ), we also note that the magnitude of $\tau$ for the $\mathrm{Ag}_{799}$

Table 3. Parameters of the Different Best-Fit Curves for the Distance Dependence of $\Delta E_{\text {blueshift }} \Delta E_{\text {redshift }}$ or $\Delta \lambda / \lambda_{0}$ for Dimer A and Dimer B Assemblies of Silver Nanorods

\begin{tabular}{|c|c|c|c|c|c|c|c|c|c|}
\hline \multirow[b]{3}{*}{ system } & \multicolumn{6}{|c|}{$\Delta E_{\text {blueshift }}$ or $\Delta E_{\text {redshift }}$ vs $R$} & \multicolumn{3}{|c|}{$\Delta \lambda / \lambda_{0}$ vs $R$} \\
\hline & \multicolumn{3}{|c|}{$\Delta E=A / R^{N}$} & \multicolumn{3}{|c|}{$\Delta E=A \mathrm{e}^{-b R}$} & \multicolumn{3}{|c|}{$\Delta \lambda / \lambda_{0}=A \mathrm{e}^{(-R / L) / \tau}$} \\
\hline & $\bar{A}$ & $\bar{N}$ & $R^{2}$ & $\bar{A}$ & $b$ & $R^{2}$ & $\bar{A}$ & $\bar{\tau}$ & $R^{2}$ \\
\hline \multicolumn{10}{|l|}{ dimer $\mathrm{A}$} \\
\hline $\mathrm{Ag}_{199}$ & 0.118 & 0.848 & 0.969 & 0.204 & 0.454 & 0.988 & 0.193 & 0.247 & 0.996 \\
\hline $\mathrm{Ag}_{391}$ & 0.098 & 0.601 & 0.965 & 0.146 & 0.324 & 0.993 & 0.216 & 0.188 & 0.996 \\
\hline $\mathrm{Ag}_{799}$ & 0.068 & 0.422 & 0.972 & 0.090 & 0.226 & 0.989 & 0.231 & 0.137 & 0.993 \\
\hline \multicolumn{10}{|l|}{ dimer $B$} \\
\hline $\mathrm{Ag}_{199}$ & 0.039 & 0.858 & 0.987 & 0.066 & 0.452 & 0.975 & 0.078 & 0.228 & 0.973 \\
\hline $\mathrm{Ag}_{391}$ & 0.025 & 0.620 & 0.985 & 0.036 & 0.327 & 0.977 & 0.071 & 0.159 & 0.975 \\
\hline $\mathrm{Ag}_{799}$ & 0.013 & 0.431 & 0.985 & 0.017 & 0.228 & 0.976 & 0.060 & 0.112 & 0.975 \\
\hline
\end{tabular}


dimers is somewhat smaller than the reported values. As shown in Table 3, the predicted values of $\tau$ are strongly dependent on the length of the nanorod rather than the universal value $(\tau \sim$ 0.2 ) with no length dependence predicted initially. ${ }^{48}$ Therefore, this deviation is most likely due to the fact that the aspect ratio for this nanorod differs significantly from the previously investigated cases, where the aspect ratios were often between 2 and 4. A future investigation in our group will address the dependence of fractional shifts on the aspect ratio/width of the nanorod by employing TD-DFT and TD-DFTB.

\section{CONCLUSIONS}

In this study, we investigated the performance of TD-DFTB for the calculated optical properties of $\mathrm{Ag}$ nanorods. Our comparison between the spectra obtained from TD-DFT and TD-DFTB calculations shows that there is a good semiquantitative agreement between the two methods as the spectral shape as well as trends in energies and intensities predicted by TD-DFT calculations can be successfully reproduced with TD-DFTB. We note that both the longitudinal and transverse peak energies are underestimated with TD-DFTB compared to the peak energies obtained with TD-DFT. This energy difference does not vary much for the transverse peak and ranges from 0.94 to $1.06 \mathrm{eV}$ for the investigated systems, whereas this difference for the longitudinal peak becomes smaller in a converging manner with increasing length of the nanorod. Additionally, the intensities for both features with the TD-DFTB level of theory are systematically underestimated compared to those obtained at the TD-DFT level. However, this underestimation is quite similar for the overall spectra and has little effect on the general agreement between the two levels of theory.

The absorption spectra obtained from TD-DFT and TDDFTB calculations are compared for dimer assemblies. For both assemblies, TD-DFTB successfully produces the splitting or the relative energy shifts of the peaks observed with TDDFT. One main difference between the two methods is that the interaction between the individual nanorod energy levels are often underestimated with TD-DFTB for smaller gaps. As a result, the relative shifts or splittings of the peaks are not as pronounced with TD-DFTB compared to the spectra obtained from TD-DFT calculations.

We also performed TD-DFTB calculations for the monomer and dimers of longer nanorods $\left(\mathrm{Ag}_{91}-\mathrm{Ag}_{985}\right)$. For monomers, both longitudinal and transverse peaks gain intensity with increasing length; however, this intensity gain is significantly larger for the longitudinal peak, as expected. In both cases, the intensity increases linearly with respect to the length of the nanorod. For dimers with a short interparticle distance (0.4 $\mathrm{nm})$, the splitting or the relative shift of the longitudinal peak shows a strong dependence on the length of the nanorod. The splitting of the longitudinal peak is not as pronounced in the spectra for the dimers of longer nanorods, such as $\mathrm{Ag}_{799}$ or $\mathrm{Ag}_{985}$. In comparison, the transverse peak only shows an intensity increase with increasing length, which is likely related to the fact that the width of the investigated nanorods is constant.

We also analyzed the dependence of the blueshift or redshift of the longitudinal peak on the length of the nanorod in dimer assemblies. Our results show a strong dependence on the length of the nanorod. In all cases, the distance dependence of the energy shifts in dimers deviates significantly from the dipole-dipole interaction model. Additionally, the dependence of fractional shifts $\left(\Delta \lambda / \lambda_{0}\right)$ has been analyzed using the plasmon ruler equation. It is seen that the best-fit curves obtained from TD-DFTB results yield values that are in good agreement with previous results in the literature. However, the comparison of the best-fit curves indicates that the parameters of the plasmon ruler equation depend on the length of the nanorods and the type of the assembly.

In principle, DFTB and TD-DFTB methods can be applied to other plasmonic systems of interest, such as silver nanoclusters with different shapes, gold nanoclusters, or nanoclusters that are protected with ligands. However, in most cases, such investigations require thorough testing of the existing parameter sets or possible development of new parameter sets for these systems. We hope that our current work can provide a basis for these investigations in the future.

\section{ASSOCIATED CONTENT}

\section{Supporting Information}

The Supporting Information is available free of charge on the ACS Publications website at DOI: 10.1021/acs.jpcc.8b05196.

Coordinates of the $\mathrm{Ag}_{6}$ base unit; charge dependence of the absorption spectra for $\mathrm{Ag}_{391}$ dimers; electronic structure, density of states, and excited-state configurations for $\mathrm{Ag}_{55}$ nanorod and its dimers; orbital interactions for $\mathrm{Ag}_{55}$ dimers; calculated bonding energy and highest occupied molecular orbital-lowest unoccupied molecular orbital gap of the $\mathrm{Ag}_{2}$ system at various bond lengths with DFTB; and length dependence of longitudinal and transverse peak in monomers (PDF)

\section{AUTHOR INFORMATION}

\section{Corresponding Author}

*E-mail: cmaikens@ksu.edu. Tel: 1-785-532-0954.

ORCID $\odot$

Christine M. Aikens: 0000-0002-0854-7997

Notes

The authors declare no competing financial interest.

\section{ACKNOWLEDGMENTS}

This study is based on work supported by the Air Force Office of Scientific Research under Grant FA9550-15-0114. The computing for this project was performed on the Beocat Research Cluster at Kansas State University, which was funded in part by NSF grants CHE-1726332, CNS-1006860, EPS1006860, and EPS-0919443.

\section{REFERENCES}

(1) Yu, Y. Y.; Chang, S. S.; Lee, C. L.; Wang, C. R. C. Gold Nanorods: Electrochemical Synthesis and Optical Properties. J. Phys. Chem. B 1997, 101, 6661-6664.

(2) Link, S.; El-Sayed, M. A. Spectral Properties and Relaxation Dynamics of Surface Plasmon Electronic Oscillations in Gold and Silver Nanodots and Nanorods. J. Phys. Chem. B 1999, 103, 84108426.

(3) Jana, N. R.; Gearheart, L.; Murphy, C. J. Seed-Mediated Growth Approach for Shape-Controlled Synthesis of Spheroidal and Rod-Like Gold Nanoparticles Using a Surfactant Template. Adv. Mater. 2001, 13, 1389-1393.

(4) Burda, C.; Chen, X. B.; Narayanan, R.; El-Sayed, M. A. Chemistry and Properties of Nanocrystals of Different Shapes. Chem. Rev. 2005, $105,1025-1102$.

(5) Eustis, S.; El-Sayed, M. A. Why Gold Nanoparticles Are More Precious Than Pretty Gold: Noble Metal Surface Plasmon Resonance 
and Its Enhancement of the Radiative and Nonradiative Properties of Nanocrystals of Different Shapes. Chem. Soc. Rev. 2006, 35, 209-217.

(6) Link, S.; Mohamed, M. B.; El-Sayed, M. A. Simulation of the Optical Absorption Spectra of Gold Nanorods as a Function of Their Aspect Ratio and the Effect of the Medium Dielectric Constant. J. Phys. Chem. B 1999, 103, 3073-3077.

(7) Guidez, E. B.; Aikens, C. M. Quantum Mechanical Origin of the Plasmon: From Molecular Systems to Nanoparticles. Nanoscale 2014, 6, 11512-11527.

(8) Wang, H.; Brandl, D. W.; Nordlander, P.; Halas, N. J. Plasmonic Nanostructures: Artificial Molecules. Acc. Chem. Res. 2007, 40, 53-62.

(9) Rycenga, M.; Cobley, C. M.; Zeng, J.; Li, W. Y.; Moran, C. H.; Zhang, Q.; Qin, D.; Xia, Y. N. Controlling the Synthesis and Assembly of Silver Nanostructures for Plasmonic Applications. Chem. Rev. 2011, 111, 3669-3712.

(10) Chen, H. J.; Shao, L.; Li, Q.; Wang, J. F. Gold Nanorods and Their Plasmonic Properties. Chem. Soc. Rev. 2013, 42, 2679-2724.

(11) Tian, L.; Chen, E.; Gandra, N.; Abbas, A.; Singamaneni, S. Gold Nanorods as Plasmonic Nanotransducers: Distance-Dependent Refractive Index Sensitivity. Langmuir 2012, 28, 17435-17442.

(12) Duan, J. S.; Park, K.; MacCuspie, R. I.; Vaia, R. A.; Pachter, R. Optical Properties of Rodlike Metallic Nanostructures: Insight from Theory and Experiment. J. Phys. Chem. C 2009, 113, 15524-15532.

(13) Khlebtsov, B. N.; Khlebtsov, N. G. Multipole Plasmons in Metal Nanorods: Scaling Properties and Dependence on Particle Size, Shape, Orientation, and Dielectric Environment. J. Phys. Chem. C 2007, 111, 11516-11527.

(14) Gonzalez, A. L.; Noguez, C.; Ortiz, G. P.; Rodriguez-Gattorno, G. Optical Absorbance of Colloidal Suspensions of Silver Polyhedral Nanoparticles. J. Phys. Chem. B 2005, 109, 17512-17517.

(15) Near, R. D.; Hayden, S. C.; El-Sayed, M. A. Thin to Thick, Short to Long: Spectral Properties of Gold Nanorods by Theoretical Modeling. J. Phys. Chem. C 2013, 117, 18653-18656.

(16) Bae, G. T.; Aikens, C. M. Time-Dependent Density Functional Theory Studies of Optical Properties of Ag Nanoparticles: Octahedra, Truncated Octahedra, and Icosahedra. J. Phys. Chem. C 2012, 116, 10356-10367.

(17) Ma, Y.; Li, W.; Cho, E. C.; Li, Z.; Yu, T.; Zeng, J.; Xie, Z.; Xia, Y. $\mathrm{Au}$ @Ag Core-Shell Nanocubes with Finely Tuned and WellControlled Sizes, Shell Thicknesses, and Optical Properties. ACS Nano 2010, 4, 6725-6734.

(18) López-Lozano, X.; Mottet, C.; Weissker, H.-C. Effect of Alloying on the Optical Properties of Ag-Au Nanoparticles. J. Phys. Chem. C 2013, 117, 3062-3068.

(19) El-Sayed, I. H.; Huang, X. H.; El-Sayed, M. A. Surface Plasmon Resonance Scattering and Absorption of Anti-EGFR Antibody Conjugated Gold Nanoparticles in Cancer Diagnostics: Applications in Oral Cancer. Nano Lett. 2005, 5, 829-834.

(20) Huang, X.; El-Sayed, I. H.; Qian, W.; El-Sayed, M. A. Cancer Cell Imaging and Photothermal Therapy in the near-Infrared Region by Using Gold Nanorods. J. Am. Chem. Soc. 2006, 128, 2115-2120.

(21) Jain, P. K.; El-Sayed, I. H.; El-Sayed, M. A. Au Nanoparticles Target Cancer. Nano Today 2007, 2, 18-29.

(22) Jain, P. K.; Huang, X.; El-Sayed, I. H.; El-Sayad, M. A. Review of Some Interesting Surface Plasmon Resonance-Enhanced Properties of Noble Metal Nanoparticles and Their Applications to Biosystems. Plasmonics 2007, 2, 107-118.

(23) Yu, C.; Irudayaraj, J. Multiplex Biosensor Using Gold Nanorods. Anal. Chem. 2007, 79, 572-579.

(24) Sperling, R. A.; Gil, P. R.; Zhang, F.; Zanella, M.; Parak, W. J. Biological Applications of Gold Nanoparticles. Chem. Soc. Rev. 2008, 37, 1896-1908.

(25) Huang, X.; Neretina, S.; El-Sayed, M. A. Gold Nanorods: From Synthesis and Properties to Biological and Biomedical Applications. Adv. Mater. 2009, 21, 4880-4910.

(26) Xu, L. G.; Kuang, H.; Wang, L. B.; Xu, C. L. Gold Nanorod Ensembles as Artificial Molecules for Applications in Sensors. J. Mater. Chem. 2011, 21, 16759-16782.
(27) Zhu, Y.; Qian, H.; Jin, R. Catalysis Opportunities of Atomically Precise Gold Nanoclusters. J. Mater. Chem. 2011, 21, 6793-6799.

(28) Stratakis, M.; Garcia, H. Catalysis by Supported Gold Nanoparticles: Beyond Aerobic Oxidative Processes. Chem. Rev. 2012, 112, 4469-4506.

(29) Austin, L. A.; Mackey, M. A.; Dreaden, E. C.; El-Sayed, M. A. The Optical, Photothermal, and Facile Surface Chemical Properties of Gold and Silver Nanoparticles in Biodiagnostics, Therapy, and Drug Delivery. Arch. Toxicol. 2014, 88, 1391-1417.

(30) Zhang, Y.; Chu, W.; Foroushani, A. D.; Wang, H.; Li, D.; Liu, J.; Barrow, C. J.; Wang, X.; Yang, W. New Gold Nanostructures for Sensor Applications: A Review. Materials 2014, 7, 5169-5201.

(31) Yang, X.; Yang, M.; Pang, B.; Vara, M.; Xia, Y. Gold Nanomaterials at Work in Biomedicine. Chem. Rev. 2015, 115, 10410-10488.

(32) Ozbay, E. Plasmonics: Merging Photonics and Electronics at Nanoscale Dimensions. Science 2006, 311, 189-193.

(33) Prodan, E.; Radloff, C.; Halas, N. J.; Nordlander, P. A Hybridization Model for the Plasmon Response of Complex Nanostructures. Science 2003, 302, 419-422.

(34) Rechberger, W.; Hohenau, A.; Leitner, A.; Krenn, J. R.; Lamprecht, B.; Aussenegg, F. R. Optical Properties of Two Interacting Gold Nanoparticles. Opt. Commun. 2003, 220, 137-141.

(35) Su, K. H.; Wei, Q. H.; Zhang, X.; Mock, J. J.; Smith, D. R.; Schultz, S. Interparticle Coupling Effects on Plasmon Resonances of Nanogold Particles. Nano Lett. 2003, 3, 1087-1090.

(36) Atay, T.; Song, J. H.; Nurmikko, A. V. Strongly Interacting Plasmon Nanoparticle Pairs: From Dipole-Dipole Interaction to Conductively Coupled Regime. Nano Lett. 2004, 4, 1627-1631.

(37) Nordlander, P.; Oubre, C.; Prodan, E.; Li, K.; Stockman, M. I. Plasmon Hybridizaton in Nanoparticle Dimers. Nano Lett. 2004, 4, 899-903.

(38) Jain, P. K.; Eustis, S.; El-Sayed, M. A. Plasmon Coupling in Nanorod Assemblies: Optical Absorption, Discrete Dipole Approximation Simulation, and Exciton-Coupling Model. J. Phys. Chem. B 2006, 110, 18243-18253.

(39) Jain, P. K.; Huang, W.; El-Sayed, M. A. On the Universal Scaling Behavior of the Distance Decay of Plasmon Coupling in Metal Nanoparticle Pairs: A Plasmon Ruler Equation. Nano Lett. 2007, 7, 2080-2088.

(40) Fofang, N. T.; Park, T. H.; Neumann, O.; Mirin, N. A.; Nordlander, P.; Halas, N. J. Plexcitonic Nanoparticles: PlasmonExciton Coupling in Nanoshell-J-Aggregate Complexes. Nano Lett. 2008, 8, 3481-3487.

(41) Jain, P. K.; El-Sayed, M. A. Noble Metal Nanoparticle Pairs: Effect of Medium for Enhanced Nanosensing. Nano Lett. 2008, 8, 4347-4352.

(42) Aćimović, S. S.; Kreuzer, M. P.; González, M. U.; Quidant, R. Plasmon near-Field Coupling in Metal Dimers as a Step toward SingleMolecule Sensing. ACS Nano 2009, 3, 1231-1237.

(43) Funston, A. M.; Novo, C.; Davis, T. J.; Mulvaney, P. Plasmon Coupling of Gold Nanorods at Short Distances and in Different Geometries. Nano Lett. 2009, 9, 1651-1658.

(44) Tabor, C.; Van Haute, D.; El-Sayed, M. A. Effect of Orientation on Plasmonic Coupling between Gold Nanorods. ACS Nano 2009, 3, $3670-3678$

(45) Zuloaga, J.; Prodan, E.; Nordlander, P. Quantum Description of the Plasmon Resonances of a Nanoparticle Dimer. Nano Lett. 2009, 9, $887-891$.

(46) Huang, C. P.; Yin, X. G.; Kong, L. B.; Zhu, Y. Y. Interactions of Nanorod Particles in the Strong Coupling Regime. J. Phys. Chem. C 2010, 114, 21123-21131.

(47) Sun, Z.; Ni, W.; Yang, Z.; Kou, X.; Li, L.; Wang, J. PhControlled Reversible Assembly and Disassembly of Gold Nanorods. Small 2008, 4, 1287-1292.

(48) Jain, P. K.; El-Sayed, M. A. Plasmonic Coupling in Noble Metal Nanostructures. Chem. Phys. Lett. 2010, 487, 153-164. 
(49) Shao, L.; Woo, K. C.; Chen, H. J.; Jin, Z.; Wang, J. F.; Lin, H. Q. Angle- and Energy-Resolved Plasmon Coupling in Gold Nanorod Dimers. ACS Nano 2010, 4, 3053-3062.

(50) Kelly, K. L.; Coronado, E.; Zhao, L. L.; Schatz, G. C. The Optical Properties of Metal Nanoparticles: The Influence of Size, Shape, and Dielectric Environment. J. Phys. Chem. B 2003, 107, 668677.

(51) Myroshnychenko, V.; Rodriguez-Fernandez, J.; Pastoriza-Santos, I.; Funston, A. M.; Novo, C.; Mulvaney, P.; Liz-Marzan, L. M.; García de Abajo, F. J. Modelling the Optical Response of Gold Nanoparticles. Chem. Soc. Rev. 2008, 37, 1792-1805.

(52) Jain, P. K.; Lee, K. S.; El-Sayed, I. H.; El-Sayed, M. A. Calculated Absorption and Scattering Properties of Gold Nanoparticles of Different Size, Shape, and Composition: Applications in Biological Imaging and Biomedicine. J. Phys. Chem. B 2006, 110, 7238-7248.

(53) Wu, Y. P.; Nordlander, P. Finite-Difference Time-Domain Modeling of the Optical Properties of Nanoparticles near Dielectric Substrates. J. Phys. Chem. C 2010, 114, 7302-7307.

(54) Oubre, C.; Nordlander, P. Optical Properties of Metallodielectric Nanostructures Calculated Using the Finite Difference Time Domain Method. J. Phys. Chem. B 2004, 108, 17740-17747.

(55) Sinha-Roy, R.; Garcia-Gonzalez, P.; Weissker, H. C.; Rabilloud, F.; Fernandez-Dominguez, A. I. Classical and Ab Initio Plasmonics Meet at Sub-Nanometric Noble Metal Rods. ACS Photonics 2017, 4, 1484-1493.

(56) McMahon, J. M.; Gray, S. K.; Schatz, G. C. Nonlocal Optical Response of Metal Nanostructures with Arbitrary Shape. Phys. Rev. Lett. 2009, 103, No. 097403.

(57) Esteban, R.; Borisov, A. G.; Nordlander, P.; Aizpurua, J. Bridging Quantum and Classical Plasmonics with a Quantum-Corrected Model. Nat. Commun. 2012, 3, No. 825.

(58) Zhu, W.; Esteban, R.; Borisov, A. G.; Baumberg, J. J.; Nordlander, P.; Lezec, H. J.; Aizpurua, J.; Crozier, K. B. Quantum Mechanical Effects in Plasmonic Structures with Subnanometre Gaps. Nat. Commun. 2016, 7, No. 11495.

(59) Morton, S. M.; Silverstein, D. W.; Jensen, L. Theoretical Studies of Plasmonics Using Electronic Structure Methods. Chem. Rev. 2011, 111, 3962-3994.

(60) Aikens, C. M.; Li, S. Z.; Schatz, G. C. From Discrete Electronic States to Plasmons: TDDFT Optical Absorption Properties of $\mathrm{Ag}_{n}(n$ $=10,20,35,56,84,120)$ Tetrahedral Clusters. J. Phys. Chem. C 2008, 112, 11272-11279.

(61) Johnson, H. E.; Aikens, C. A. Electronic Structure and TDDFT Optical Absorption Spectra of Silver Nanorods. J. Phys. Chem. A 2009, $113,4445-4450$.

(62) Bae, G. T.; Aikens, C. M. TDDFT and CIS Studies of Optical Properties of Dimers of Silver Tetrahedra. J. Phys. Chem. A 2012, 116, $8260-8269$.

(63) Guidez, E. B.; Aikens, C. M. Diameter Dependence of the Excitation Spectra of Silver and Gold Nanorods. J. Phys. Chem. C 2013, 117, 12325-12336.

(64) Vincenot, J.; Aikens, C. M. Quantum Mechanical Examination of Optical Absorption Spectra of Silver Nanorod Dimers. In Advances in the Theory of Atomic and Molecular Systems; Piecuch, P., Maruani, J., Delgado-Barrio, G., Wilson, S., Eds.; Springer: Netherlands, 2009; Vol. 20, pp 253-264.

(65) Stener, M.; Nardelli, A.; De Francesco, R.; Fronzoni, G. Optical Excitations of Gold Nanoparticles: A Quantum Chemical Scalar Relativistic Time Dependent Density Functional Study. J. Phys. Chem. C 2007, 111, 11862-11871.

(66) Durante, N.; Fortunelli, A.; Broyer, M.; Stener, M. Optical Properties of Au Nanoclusters from TD-DFT Calculations. J. Phys. Chem. C 2011, 115, 6277-6282.

(67) Barcaro, G.; Sernenta, L.; Fortunelli, A.; Stener, M. Optical Properties of Silver Nanoshells from Time-Dependent Density Functional Theory Calculations. J. Phys. Chem. C 2014, 118, $12450-12458$.
(68) Weissker, H. C.; Mottet, C. Optical Properties of Pure and Core-Shell Noble-Metal Nanoclusters from TDDFT: The Influence of the Atomic Structure. Phys. Rev. B 2011, 84, No. 165443.

(69) López-Lozano, X.; Barron, H.; Mottet, C.; Weissker, H.-C. Aspect-Ratio- and Size-Dependent Emergence of the Surface-Plasmon Resonance in Gold Nanorods - an Ab Initio TDDFT Study. Phys. Chem. Chem. Phys. 2014, 16, 1820-1823.

(70) Liao, M. S.; Bonifassi, P.; Leszczynski, J.; Ray, P. C.; Huang, M. J.; Watts, J. D. Structure, Bonding, and Linear Optical Properties of a Series of Silver and Gold Nanorod Clusters: DFT/TDDFT Studies. J. Phys. Chem. A 2010, 114, 12701-12708.

(71) Iida, K.; Noda, M.; Ishimura, K.; Nobusada, K. First-Principles Computational Visualization of Localized Surface Plasmon Resonance in Gold Nanoclusters. J. Phys. Chem. A 2014, 118, 11317-11322.

(72) Grimme, S. A Simplified Tamm-Dancoff Density Functional Approach for the Electronic Excitation Spectra of Very Large Molecules. J. Chem. Phys. 2013, 138, No. 244104.

(73) Bannwarth, C.; Grimme, S. A Simplified Time-Dependent Density Functional Theory Approach for Electronic Ultraviolet and Circular Dichroism Spectra of Very Large Molecules. Comput. Theor. Chem. 2014, 1040-1041, 45-53.

(74) Prodan, E.; Nordlander, P. Structural Tunability of the Plasmon Resonances in Metallic Nanoshells. Nano Lett. 2003, 3, 543-547.

(75) Teperik, T. V.; Nordlander, P.; Aizpurua, J.; Borisov, A. G. Quantum Effects and Nonlocality in Strongly Coupled Plasmonic Nanowire Dimers. Opt. Express 2013, 21, 27306-27325.

(76) Marinica, D. C.; Kazansky, A. K.; Nordlander, P.; Aizpurua, J.; Borisov, A. G. Quantum Plasmonics: Nonlinear Effects in the Field Enhancement of a Plasmonic Nanoparticle Dimer. Nano Lett. 2012, 12, 1333-1339.

(77) Townsend, E.; Bryant, G. W. Plasmonic Properties of Metallic Nanoparticles: The Effects of Size Quantization. Nano Lett. 2012, 12, 429-434.

(78) Ekardt, W. Collective Multipole Excitations in Small Metal Particles: Critical Angular Momentum $l^{\text {ex }}$ for the Existence of Collective Surface Modes. Phys. Rev. B 1985, 32, 1961-1970.

(79) Akimov, A. V.; Prezhdo, O. V. Large-Scale Computations in Chemistry: A Bird's Eye View of a Vibrant Field. Chem. Rev. 2015, 115, 5797-5890.

(80) Varas, A.; Garcia-Gonzalez, P.; Feist, J.; Garcia-Vidal, F. J.; Rubio, A. Quantum Plasmonics: From Jellium Models to Ab Initio Calculations. Nanophotonics 2016, 5, 409-426.

(81) Fernando, A.; Weerawardene, K. L. D. M.; Karimova, N. V.; Aikens, C. M. Quantum Mechanical Studies of Large Metal, Metal Oxide, and Metal Chalcogenide Nanoparticles and Clusters. Chem. Rev. 2015, 115, 6112-6216.

(82) Porezag, D.; Frauenheim, T.; Kohler, T.; Seifert, G.; Kaschner, R. Construction of Tight-Binding-Like Potentials on the Basis of Density-Functional Theory - Application to Carbon. Phys. Rev. B 1995, 51, 12947-12957.

(83) Seifert, G.; Porezag, D.; Frauenheim, T. Calculations of Molecules, Clusters, and Solids with a Simplified LCAO-DFT-LDA Scheme. Int. J. Quantum Chem. 1996, 58, 185-192.

(84) Niehaus, T. A.; Suhai, S.; Sala, F. D.; Lugli, P.; Elstner, M.; Seifert, G.; Frauenheim, T. Tight-Binding Approach to TimeDependent Density-Functional Response Theory. Phys. Rev. B 2001, 63, No. 085108.

(85) Frauenheim, T.; Seifert, G.; Elstner, M.; Niehaus, T.; Kohler, C.; Amkreutz, M.; Sternberg, M.; Hajnal, Z.; Di Carlo, A.; Suhai, S. Atomistic Simulations of Complex Materials: Ground-State and Excited-State Properties. J. Phys.: Condens. Matter 2002, 14, 30153047.

(86) Joswig, J. O.; Seifert, G.; Niehaus, T. A.; Springborg, M. Optical Properties of Cadmium Sulfide Clusters. J. Phys. Chem. B 2003, 107, 2897-2902.

(87) Frenzel, J.; Joswig, J. O.; Sarkar, P.; Seifert, G.; Springborg, M. The Effects of Organisation, Embedding and Surfactants on the Properties of Cadmium Chalcogenide (CdS, CdSe and CdS/CdSe) Semiconductor Nanoparticles. Eur. J. Inorg. Chem. 2005, 3585-3596. 
(88) Frenzel, J.; Joswig, J. O.; Seifert, G. Optical Excitations in Cadmium Sulfide Nanoparticles. J. Phys. Chem. C 2007, 111, 1076110770.

(89) Li, Q. S.; Zhang, R. Q.; Niehaus, T. A.; Frauenheim, T.; Lee, S. T. Theoretical Studies on Optical and Electronic Properties of Propionic-Acid-Terminated Silicon Quantum Dots. J. Chem. Theory Comput. 2007, 3, 1518-1526.

(90) Wang, X.; Zhang, R. Q.; Lee, S. T.; Niehaus, T. A.; Frauenheim, T. Unusual Size Dependence of the Optical Emission Gap in Small Hydrogenated Silicon Nanoparticles. Appl. Phys. Lett. 2007, 90, No. 123116

(91) Wang, X.; Zhang, R. Q.; Niehaus, T. A.; Frauenheim, T. Excited State Properties of Allylamine-Capped Silicon Quantum Dots. J. Phys. Chem. C 2007, 111, 2394-2400.

(92) Wang, Y.; Zhang, R. Q.; Frauenheim, T.; Niehaus, T. A. Atomistic Simulations of Self-Trapped Exciton Formation in Silicon Nanostructures: The Transition from Quantum Dots to Nanowires. J. Phys. Chem. C 2009, 113, 12935-12938.

(93) Xie, R. H.; Bryant, G. W.; Sun, G. Y.; Nicklaus, M. C.; Heringer, D.; Frauenheim, T.; Manaa, M. R.; Smith, V. H.; Araki, Y.; Ito, O. Excitations, Optical Absorption Spectra, and Optical Excitonic Gaps of Heterofullerenes. 1. $\mathrm{C}^{60}, \mathrm{C}^{59} \mathrm{~N}^{+}$, and $\mathrm{C}^{48} \mathrm{~N}^{12}$ : Theory and Experiment. J. Chem. Phys. 2004, 120, 5133-5147.

(94) Wendumu, T. B.; Seifert, G.; Lorenz, T.; Joswig, J. O.; Enyashin, A. Optical Properties of Triangular Molybdenum Disulfide Nanoflakes. J. Phys. Chem. Lett. 2014, 5, 3636-3640.

(95) Fan, G. H.; Zhu, S.; Xu, H. Density-Functional Theory Study of the Interaction Mechanism and Optical Properties of Flavonols on the Boron Nitride Nanotubes. Int. J. Quantum Chem. 2018, 118, No. e25514.

(96) Douglas-Gallardo, O. A.; Berdakin, M.; Sanchez, C. G. Atomistic Insights into Chemical Interface Damping of Surface Plasmon Excitations in Silver Nanoclusters. J. Phys. Chem. C 2016, 120, 24389-24399.

(97) Bonafé, F. P.; Aradi, B.; Guan, M. X.; Douglas-Gallardo, O. A.; Lian, C.; Meng, S.; Frauenheim, T.; Sánchez, C. G. Plasmon-Driven Sub-Picosecond Breathing of Metal Nanoparticles. Nanoscale 2017, 9, 12391-12397.

(98) Douglas-Gallardo, O. A.; Soldano, G. J.; Mariscal, M. M.; Sanchez, C. G. Effects of Oxidation on the Plasmonic Properties of Aluminum Nanoclusters. Nanoscale 2017, 9, 17471-17480.

(99) Ilawe, N. V.; Oviedo, M. B.; Wong, B. M. Real-Time Quantum Dynamics of Long-Range Electronic Excitation Transfer in Plasmonic Nanoantennas. J. Chem. Theory Comput. 2017, 13, 3442-3454.

(100) ADF. SCM, Theoretical Chemistry; Vrije Universiteit: Amsterdam, The Netherlands, 2016. http://www.scm.com/.

(101) Guerra, C. F.; Snijders, J. G.; te Velde, G.; Baerends, E. J. Towards an Order-N DFT Method. Theor. Chem. Acc. 1998, 99, 391403.

(102) te Velde, G.; Bickelhaupt, F. M.; Baerends, E. J.; Guerra, C. F.; Van Gisbergen, S. J. A.; Snijders, J. G.; Ziegler, T. Chemistry with ADF. J. Comput. Chem. 2001, 22, 931-967.

(103) Perdew, J. P. Density-Functional Approximation for the Correlation-Energy of the Inhomogeneous Electron-Gas. Phys. Rev. B 1986, 33, 8822-8824.

(104) Becke, A. D. Density-Functional Exchange-Energy Approximation with Correct Asymptotic-Behavior. Phys. Rev. A 1988, 38, 3098-3100.

(105) van Gisbergen, S. J. A.; Snijders, J. G.; Baerends, E. J. Implementation of Time-Dependent Density Functional Response Equations. Comput. Phys. Commun. 1999, 118, 119-138.

(106) Rüger, R.; van Lenthe, E.; Lu, Y.; Frenzel, J.; Heine, T.; Visscher, L. Efficient Calculation of Electronic Absorption Spectra by Means of Intensity-Selected Time-Dependent Density Functional Tight Binding. J. Chem. Theory Comput. 2015, 11, 157-167.

(107) Perdew, J. P.; Burke, K.; Ernzerhof, M. Generalized Gradient Approximation Made Simple. Phys. Rev. Lett. 1996, 77, 3865-3868.
(108) Perdew, J. P.; Burke, K.; Wang, Y. Generalized Gradient Approximation for the Exchange-Correlation Hole of a Many-Electron System. Phys. Rev. B 1996, 54, 16533-16539.

(109) Elstner, M.; Porezag, D.; Jungnickel, G.; Elsner, J.; Haugk, M.; Frauenheim, T.; Suhai, S.; Seifert, G. Self-Consistent-Charge DensityFunctional Tight-Binding Method for Simulations of Complex Materials Properties. Phys. Rev. B 1998, 58, 7260-7268.

(110) Elstner, M.; Frauenheim, T.; Kaxiras, E.; Seifert, G.; Suhai, S. A Self-Consistent Charge Density-Functional Based Tight-Binding Scheme for Large Biomolecules. Phys. Status Solidi B 2000, 217, 357-376.

(111) Szűcs, B.; Hajnal, Z.; Frauenheim, T.; Gonzalez, C.; Ortega, J.; Perez, R.; Flores, F. Chalcogen Passivation of GaAs(100) Surfaces: Theoretical Study. Appl. Surf. Sci. 2003, 212-213, 861-865.

(112) Szűcs, B.; Hajnal, Z.; Scholz, R.; Sanna, S.; Frauenheim, T. Theoretical Study of the Adsorption of a PTCDA Monolayer on SPassivated GaAs(100). Appl. Surf. Sci. 2004, 234, 173-177.

(113) Willingham, B.; Brandl, D. W.; Nordlander, P. Plasmon Hybridization in Nanorod Dimers. Appl. Phys. B 2008, 93, 209-216.

(114) Dolinnyi, A. I. Nanometric Rulers Based on Plasmon Coupling in Pairs of Gold Nanoparticles. J. Phys. Chem. C 2015, 119, 49905001. 